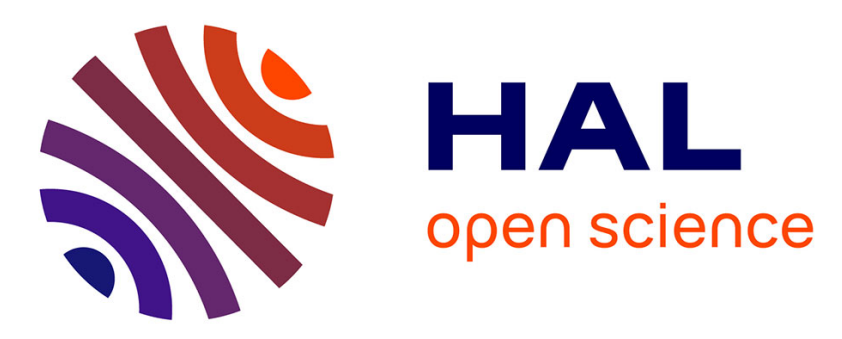

\title{
Ecodesign of Ordered Mesoporous Materials Obtained with Switchable Micellar Assemblies
}

Niki Baccile, Julien Reboul, Béatrice Blanc, Bernard Coq, Patrick

Lacroix-Desmazes, Martin In, Corine Gerardin

\section{- To cite this version:}

Niki Baccile, Julien Reboul, Béatrice Blanc, Bernard Coq, Patrick Lacroix-Desmazes, et al.. Ecodesign of Ordered Mesoporous Materials Obtained with Switchable Micellar Assemblies. Angewandte Chemie International Edition, 2008, 47, pp.8433-8437. 10.1002/anie.200802431 . hal-00340794

\author{
HAL Id: hal-00340794 \\ https://hal.science/hal-00340794
}

Submitted on 14 Feb 2017

HAL is a multi-disciplinary open access archive for the deposit and dissemination of scientific research documents, whether they are published or not. The documents may come from teaching and research institutions in France or abroad, or from public or private research centers.
L'archive ouverte pluridisciplinaire HAL, est destinée au dépôt et à la diffusion de documents scientifiques de niveau recherche, publiés ou non, émanant des établissements d'enseignement et de recherche français ou étrangers, des laboratoires publics ou privés. 
IMPORTANT NOTE: Please be aware that slight modifications occurring after Proof correction may occur between this version of the manuscript and the version on the Publisher's website-

\section{Ecodesign of Ordered Mesoporous Materials Obtained with Switchable Micellar Assemblies**.}

Niki Baccile, Julien Reboul, Béatrice Blanc, Bernard Coq, Patrick Lacroix-Desmazes, Martin In, Corine Gérardin ${ }^{*}$.

N. Baccile, J. Reboul, B. Blanc, B. Coq, P. Lacroix-Desmazes, C. Gérardin Institut Charles Gerhardt Montpellier - UMR 5253 CNRS/ENSCM/UM2/UM1, 8, rue de l'Ecole Normale - 34296 Montpellier cedex 5 - France.

M. In Laboratoire des Colloïdes, Verres et Nanomatériaux, Université Montpellier 2, 34095 Montpellier cedex 5 - France.

N. Baccile Present address: Max-Planck Institute for Colloids and Interfaces, Am Mühlenberg 1, 14476, Golm, Germany

* C. Gérardin

* to whom correspondence should be sent. Fax : 33 (0) 4671634 70, corine.gerardin@enscm.fr

[**] Authors thank P. Dieudonné (University Montpellier 2, France) for SAXS measurements and F. Babonneau and J. Maquet (University P. M. Curie, France) for solid-state NMR experiments. They also thank H. Amenitsch (IBN, Graz, Austria) and Plinio Innocenzi (University of Sassari, Italy) for their help in SAXS data treatment, and T. Nugay (Bogazici University, Turkey) for his help in polymer synthesis. G. Calleja (ICG Montpellier, France) is acknowledged for artwork. This work was supported by the French Minister of Education, Research and Technology in the frame of the ACI Project JC 4092. 


\begin{abstract}
The synergetic coupling between sol-gel chemistry and supramolecular assemblies of amphiphilic molecules results in ordered nanostructured hybrid materials. These hybrid nanostructures are precursors of the so-called mesoporous materials exhibiting highly ordered porosity useful in separation, catalysis, drug release. In the past decade, a large set of functional mesoporous materials shaped as monoliths, thin films or powders have been synthesized. However, many of them remain within the academic field because some of their limitations effectively close the door to the real market, among them: cost, unsustainable solvents, energyconsuming process, toxicity of some structure-directing agents and their non-recyclability. Herein, we describe an innovative green strategy for preparing ordered mesoporous materials in water at room temperature, allowing an eco-recycling of the template. We use, as new structuring agents, water-soluble block copolymers whose micellar assembly can be reversibly tuned and triggered in water by physico-chemical parameters $(\mathrm{pH}$, temperature...). The possible disassembling of the embedded "smart" micelles in aqueous solution generates porosity and releases the micelle components for further structuring runs. This report paves the way to the one-pot synthesis of ordered mesoporous materials under sustainable conditions. The synthesis process of nanostructured inorganic materials can be developed in water as a continuous process using recyclable structuring agents.
\end{abstract}


The synthesis of long-range ordered nanoscale structured materials and ordered mesoporous materials (OMM) was strongly developed in the past 15 years due to the exploitation of amphiphilic properties of some molecules ${ }^{[1,2,3,4]}$ with a lyotropic character and behaving as structuring directing agents (SDA). The success of these materials is due to their easy shaping (powders, thin films, monoliths), the wide chemical compositions of the inorganic frameworks and, after removal of the SDA, the high specific surface areas, as well as the controlled pore size distribution and geometry ${ }^{[5]}$. Easy surface modification also contributed to their success. These features have made them very interesting materials for applications in adsorption, catalysis, sensing or as host materials for drugs ${ }^{[6]}$.

In spite of several hundreds of recent reports, which emphasize the fascinating potentialities of OMM, the implementation at a large-scale production remains limited. The first and main reason stands in the production cost of the porous materials associated with the current synthesis developed until now. It was indeed recently shown in the final report of a EU funded project ${ }^{[7]}$ that over $80 \%$ of the final cost of OMM comes from the energy demand associated with the calcination step of the structure directing agent (SDA) for porosity liberation, the SDA being moreover lost. For this reason, washing in organic solvents (methanol or ethanol) ${ }^{[8]}$ and very seldom in water at reflux has become an interesting alternative route. Nevertheless, the efficiency of the process depends on the used SDA ${ }^{[9,10]}$. Alternative physical methods (use of ozone, $\mathrm{CO}_{2}$ extraction) have been proposed ${ }^{[11,12,13]}$ but the required equipment and effort overwhelm the real benefits. In all cases, a multi-step approach from the synthesis of the material to the creation of open porosity is always followed. 
A question arises: is there any strategy allowing a one-pot process for achieving structured material synthesis and pore formation? This task is challenging and the first report where such an idea was considered appeared only recently in the field of microporous zeolite materials ${ }^{[14]}$, where covalent bonds of the single-molecule SDA were chemically broken yielding two separate smaller entities, which could then be released from the microporous structure. Recycling of the SDA after re-formation of the broken bonds was proved to be successful. Supramolecular assemblies from $\pi-\pi$ interactions were also used as SDA for the synthesis of Linde type Azeolites, but the recovery of the SDA was impossible ${ }^{[15]}$. To obtain mesoporous materials, the authors generally try to develop new ways ${ }^{[14,15]}$ of structuring solids but, in the reported studies $[16,17,18,19]$, low-cost sustainable methods are hardly proposed. In addition, there are environmental concerns over the realization of these methods, e.g. use of hot $\mathrm{HCl}$ solution, as in ref. 14.

For the first time, an eco-design for preparing ordered mesoporous materials is proposed: a one-pot process in water, at room temperature and mild $\mathrm{pH}$ is proposed here for structuring inorganic materials and creating porosity by means of smart micellar assemblies of water-soluble block copolymers. Micellization of hydrophilic block copolymers and, more specifically, double-hydrophilic block copolymers (DHBC) ${ }^{[20-29]}$ can in fact be easily triggered in water by changing simple physico-chemical parameters, like $\mathrm{pH}$, temperature or ionic strength. Furthermore, micellization can be reversible in the same aqueous synthesis batch leading to the possible disassembling of the structuring agent. The latter process leads to porosity formation in the material and it makes the building units of the SDA available for further synthesis runs. Thus, this process allows SDA recycling in water under sustainable conditions due to the non-toxicity of initial components (e.g. polysaccharides) and solvent (water) and to the low-energy interactions (electrostatic, Van der Waals) involved in the reversible micellar assembling process. The present energy-and atom-saving methodology that we propose is schematized in Figure 1. 
Hence the present report proposes three main innovating points: Firstly, ordered nanostructured materials can be prepared by using smart micellar aggregates of stimuli-responsive DHBC as new structuring agents. Secondly, the SDA can be driven out by a simple washing step in water at room temperature at a well-chosen nearly neutral $\mathrm{pH}$; porosity is then created. Thirdly, based on the two first points, a continuous one-pot process can be developed, it allows synthesizing a nanostructured material, washing to yield the corresponding mesoporous material, and recycling of the SDA in order to prepare a new porous solid within the same batch.

The feasibility of our strategy is demonstrated here by structuring silica at room temperature using reversible $\mathrm{pH}$-responding micellar aggregates. As a proof-of-concept, we prepared mesostructured silica-based materials by templating with micelles of poly(ethyleneoxide)-blockpoly(methacrylic acid), PEO-b-PMAA, or poly(ethyleneoxide)-block-poly(acrylic acid), PEO-bPAA block copolymers, and an oligochitosan lactate (OCL) as a co-micellization agent. Block copolymers are now easily available at the bench scale thanks to the development of various controlled radical polymerization methods, some of them being developed at an industrial scale. [30-32] Micellization of the present polyelectrolyte-neutral DHBCs is induced by electrostatic complexation of the oppositely charged macroion. Herein, double hydrophilic polyacid-neutral block copolymers ${ }^{[20,21,22]}$ are used in the presence of a weak polybase. Formation of an electrostatic complex between the two oppositely charged polyions leads to the formation of core-corona aggregates with a water-insoluble complex core and a water-soluble corona. Formation of the micellar aggregates can be driven by the $\mathrm{pH}$ since the charges of the polyacid and polybase blocks are pH-dependent. Micellization of DHBC [20,21,23,24,25] and more specifically, formation of polyion complex (PIC) micelles ${ }^{[26,27,28,29]}$ were extensively studied in the literature. Mixtures of PEO-b-PMAA or PEO-b-PAA block copolymers and OCL lead to PIC 
micelles with an electrostatic complex core and a PEO corona. Because the switchable assembly/disassembly process of DHBCs only involves weak interactions such as electrostatic interactions, the energy demand for disassembly is quite low in comparison with covalent bonds breaking. When a silica precursor, like tetraethoxysilane (TEOS), is added to the PIC micelle solution, a nanostructured solid is obtained at the end of the hydrolysis and condensation process of the silicon alkoxide.

The formation of micellar objects between OCL and the hydrosoluble block copolymer, as a function of $\mathrm{pH}$, was followed by dynamic light scattering (DLS). The measurement of the light scattered intensity of the DHBC/OCL mixture allowed showing that formation of micellar aggregates occurs at a pH from 4.5 to 7 . Results are shown on Figure 2 in the case of the PEO-bPAA/OCL system (with an amine/carboxylic acid molar ratio of 1). In the $\mathrm{pH}$ domain 4.5 to 7 , well-defined micelles are formed and their intensity-averaged hydrodynamic diameter equals 25 nm. Increasing the $\mathrm{pH}$ above 7.5 (by adding a $\mathrm{NaOH}$ solution) leads to a strong decrease of the scattered intensity that reveals the dissociation of the micelles; this is due to the neutralization of the amine functions of the homopolymer polybase.

Precisely, the synthesis of the hybrid material proceeds as follows: first, the DHBC and the polyamine are dissolved in an aqueous solution, then TEOS is added and the $\mathrm{pH}$ is decreased to 2 for TEOS hydrolysis, which is completed within 15 minutes. The hybrid material is then synthesized at room temperature by increasing the $\mathrm{pH}$ of the mixture to a value varying between 4.5 and 6.5 (by adding a $\mathrm{NaOH}$ solution). After 24 hours, a precipitate is recovered; it can be either filtered out and washed ex-situ in water during $48 \mathrm{~h}$ at $25^{\circ} \mathrm{C}$ at $\mathrm{pH}=8$ (corresponding to a micelle dissociating $\mathrm{pH}$ for the OCL/DHBC system) or left in its synthesis medium and the $\mathrm{pH}$ is then raised in-situ above 8 for micelle disassembly and polymer template removal from the material. The solid is then recovered and allowed to dry at $60^{\circ} \mathrm{C}$ overnight. In both cases, leftover 
solutions, expected to contain the SDA-forming polymers, are tested for a new material synthesis under the same conditions.

The possibility of structuring silica with PIC micelles is shown in Figure 3. Transmission Electron Microscopy images together with Fourier Transforms of the structures of three materials are presented: with $\mathrm{PEO}-\mathrm{b}-\mathrm{PMAA} / \mathrm{OCL}$ at $\mathrm{pH}=4.5(3 \mathrm{a})$ and $\mathrm{pH}=6.5(3 \mathrm{~b})$, and with $\mathrm{PEO}-\mathrm{b}-$ PAA/OCL at $\mathrm{pH}=5.5$ (3c). Mesostructured hybrid silica-based samples are obtained in all cases. Micelle morphology and arrangement vary from cylindrical micelles arranged in a twodimensional hexagonal structure to lamellae structures. In the PEO-b-PMAA/OCL (pH= 4.5) system, cylindrical micelles of $5 \mathrm{~nm}$ are arranged in a hexagonal pattern with a d-spacing of 12 $\mathrm{nm}$. When the synthesis $\mathrm{pH}$ is adjusted to 6.5, micellar aggregates change shapes, they adopt a lamellar morphology; the lamellae thickness is about $6 \mathrm{~nm}$. When the material synthesis was done with the PEO-b-PAA/OCL system at $\mathrm{pH}$ 5.5, a well-ordered two-dimensional hexagonal structure of cylindrical micelles is obtained (figure 3c) ; figure 3d shows the XRD pattern of the material with the three diffraction peaks ascribed to the (100), (200) and (210) planes of the hexagonal structure. The correlation distance is calculated to be $13.5 \mathrm{~nm}$. These data show that complex micelles of DHBC/oppositely charged polyelectrolytes can direct the formation of nanostructured silica in a well-chosen $\mathrm{pH}$ range. On the contrary, any attempt to obtain silica OMM in the absence of either the DHBC or the polyelectrolyte component was unsuccessful. It is also emphasized here that the synthesis $\mathrm{pH}$ has a great influence on the structure of the silica based material. Changing the synthesis $\mathrm{pH}$ from 4.5 to 6.5 allows tuning the material structure and changing from cylindrical to lamellar micelles. It should be emphasized here that a key result in this study is the evidence of the existence of PIC micelles under various morphologies: cylindrical and lamellar PIC micelles are present in the hybrid silica-containing material. The behaviour of DHBC-polyamine micelles had been thoroughly investigated in dilute aqueous 
solution, at various $\mathrm{pH}$ and ionic strength, and in the absence of silica precursors: in that conditions, only spherical PIC micelles had been put in evidence. The demonstration of the existence of different morphologies for the present PIC micelles is very important and interesting for various applications, and to the best of our knowledge, well defined cylinders and lamellae had never been observed in the case of electrostatic polyion complex micelles.

As the micelles are constituted of a polyion complex core surrounded by a PEO corona, formation of the mesostructured silica based material must be the result of hydrogen bonding between ethylene oxide units and silanols of the silica network under formation. The structuring process is then similar to the one occurring in the case of non-ionic PEO-based surfactants such as the Pluronics in the synthesis of the SBA15 ${ }^{[4]}$ type materials, for example. PIC micelles have the same interface with the solvent and with silica precursors as conventional PEO based surfactant micelles, this allows precipitation of silica/micelle mesophases and this further implies that PIC micelles do not dissociate under the material synthesis conditions. Finally, it is known that the driving force for PIC micelle core formation is the decrease of the total electrostatic free energy of the system, which includes the mixing entropy gain from the release of polymer counter-ions in solution, whereas in classical amphiphilic systems, hydrophobic interactions drive the formation of the micelle core. The properties of these attractive interactions, their response to the physico-chemical characteristics of the medium, are exploited for driving the dissociation of the micelles inside the material.

The second main point of our demonstration is the following: the ex situ washing step of the mesostructured hybrid material in aqueous solution at $\mathrm{pH} 8$ is efficient for forming the porosity; this is shown by the $\mathrm{N}_{2}$ adsorption/desorption isotherms presented in Figure 4 in the case of the PEO-b-PAA/OCL-silica system. Isotherms of the material washed in water at pH 8 
show a hysteresis loop in the range $0.6<\mathrm{P} / \mathrm{P}_{0}<0.9$, as expected for mesoporous materials, and the final apparent BET surface area amounts to $250 \mathrm{~m}^{2} / \mathrm{g}$. The pore size distribution is centered at $8.5 \mathrm{~nm}$, as determined from the desorption isotherm. This average pore size is smaller than the hydrodynamic diameter $\mathrm{Dh}$ of the micelle (25 nm), which is expected since $\mathrm{Dh}$ includes the core size (PAA/OCL complex) and the size of the large and hydrated POE corona in a good solvent in water. The efficiency of the removal of the organic structuring agent in the washed sample is quantified by comparing its thermogravimetry analysis to the one of the as-synthesized sample. As a preliminary result, a single washing step at $\mathrm{pH}=8$ allows recovering about $62 \%$ of organic matter in the case of the PEO-b-PAA/OCL system. For sake of comparison, textural properties were also examined on the unwashed PEO-b-PAA/OCL sample for which the porosity was created by a classical calcination step at $823 \mathrm{~K}$ (Figure 4): a BET surface area of $340 \mathrm{~m}^{2} / \mathrm{g}$ was obtained, with a mean pore size of $5.5 \mathrm{~nm}$. When varying the polymer characteristics, specific $\mathrm{N}_{2}$ adsorbed volumes range from 0.3 to $0.6 \mathrm{ml} / \mathrm{g}$ while BET specific surface areas vary between 110 and $740 \mathrm{~m}^{2} / \mathrm{g}$. Porosity characteristics are given in Supporting Information for different porous materials obtained by washing at room temperature, on one hand, and by calcination, on the other hand (Supplementary Figure 1).

Finally, the third new feature is the possibility of a continuous one-pot material synthesis with recycling of the SDA and restructuring of a new material. It is presented hereafter, in Figure 5 , in the case of materials synthesized at $\mathrm{pH}=4.5$ with PEO-b-PMAA and OCL polymers. It is shown that it is possible to perform multiple in situ synthesis runs. First, sample S1 was synthesized at $\mathrm{pH}$ 4.5, it was then separated by filtration, without any washing step, and recovered. The leftover supernatant solution was directly re-used for a new synthesis step by addition of TEOS, and it gave a new material, S1R. On the other hand, the same synthesis of material S1 was performed again, but this time the $\mathrm{pH}$ of the synthesis medium was increased up 
to 8 after powder precipitation in order to yield the washed material, S1w; S1w was then separated and collected. The leftover solution was reused for a new similar synthesis procedure by adding fresh TEOS, yielding the material S1wR, after synthesis and washing.

TEM images with respective Fourier Transforms and SAXS patterns show that mesostructured silica was obtained for both S1 and S1w samples, as expected, presenting an ordered micellar arrangement with inter-micellar distances of $\mathrm{d}=11.6$ and $13.4 \mathrm{~nm}$, respectively. Interestingly, S1R, which is prepared by using the supernatant solution of the non-washed S1 sample, does not exhibit any nanostructure (TEM image and SAXS pattern of S1R are shown in Supplementary Figure 2). In contrast, in the case of S1wR sample, a mesostructure is clearly identified and a long-range inter-micellar correlation distance of $12.3 \mathrm{~nm}$ is obtained. There is no change in the ordering degree of the second material, prepared from recycled polymers, compared to the first material, provided that the second synthesis is run under the same synthesis conditions. Consequently, the washing step at $\mathrm{pH}=8$ of sample $\mathrm{S} 1$ leading to $\mathrm{S} 1 \mathrm{w}$ material indeed diassembles the micellar objects and releases PEO-b-PMAA and OCL in solution. The latter are then able to act as templates again; they allow a new synthesis of mesoporous material to occur, after re-assembly of the SDA constituents at the desired pH.

${ }^{29} \mathrm{Si}$ Magic Angle Spinning (MAS) Nuclear Magnetic Resonance (NMR) experiments (Supplementary Figure 3) on as-synthesized sample S1 show a percentage of fully condensed silica sites $\mathrm{Si}(\mathrm{OSi})_{4}\left(\mathrm{Q}_{4}, \delta=-110 \mathrm{ppm}\right)$ of about $42 \%$, corresponding to an overall silica condensation degree of 0.83. In situ treatment at $\mathrm{pH}=8$ leads to an increase of the fraction of $\mathrm{Q}_{4}$ sites up to 65\% (in S1w and in S1wR), which enhances silica condensation degree up to 0.90 . Hence, washing at $\mathrm{pH}=8$ reveals to be interesting for two reasons. First, the condensation degree is comparable to materials usually obtained after a hydrothermal treatment at $100^{\circ} \mathrm{C}$, a process 
which is usually used to strengthen silica network. Secondly, no calcination step is needed to create porosity. In both cases, the saving in energy and matter can be significant.

In conclusion, we described here an innovative strategy to prepare mesoporous materials at room temperature in aqueous medium by using induced and reversible micelles of DHBCs as structuring agents. The new templates are formed by an induced micellization process in water, which can be triggered back and forth by adjusting the $\mathrm{pH}$ of the aqueous medium. This makes it possible to dissociate the template and remove it from the inorganic network in soft conditions with the objective to be recycled. We have shown that by using polyion complex micelles between a weak polybase and a polyacid-poly(ethylene oxide) DHBC, different well-ordered mesostructures can be obtained by tuning the physico-chemical parameters ( $\mathrm{pH}$, temperature) and by changing the composition of the system. Specific surface areas of the mesoporous materials obtained by room temperature washing vary from 70 to $500 \mathrm{~m}^{2} / \mathrm{g}$, depending on the synthesis conditions. Finally, we have opened the way to an in situ multiple templating process of silica in water, without the use of organic solvents. Treating the material in the synthesis aqueous medium at mildly basic $\mathrm{pH}$ firstly enhances silica condensation degree and secondly allows recycling of the templating agent, which is re-used in the same reaction medium to perform a new synthesis run. The strategy presented here was developed in the case of macroion electrostatic complex micelles for which the micellization process was triggered by the $\mathrm{pH}$, but it can be extended to other triggers: at present, we are testing the silica structuring and SDA recycling processes with micelles of DHBC whose formation is induced in water by a change of either ionic strength or temperature, the latter providing a good solution for increasing the number of cycles. Finally, the approach can also be extended to various other metal oxide-based materials. 


\section{Experimental section}

\section{Preparation of mesoporous materials}

Employed DHBC is poly(ethyleneoxide)-b-poly(methacrylic acid), PEO-b-PMAA ( $M_{P E O}=5000$ $\mathrm{g} / \mathrm{mol} ; M_{P M A A}=2100 \mathrm{~g} / \mathrm{mol}$ ) from Polymer Source, while the polyamine is oligochitosan lactate, OCL, (Aldrich) with $M<5000 \mathrm{~g} / \mathrm{mol}$. Silica precursor is tetraethylorthosilicate, TEOS (Aldrich). Typical synthesis molar ratios are TEOS/PEO-b-PMAA/H $\mathrm{H}_{2} \mathrm{O} / \mathrm{OCL}=1 / 0.00862 / 370 / 0.10678$ with OCL corresponding to an oligochitosan lactate unit $\left(\mathrm{C}_{12} \mathrm{H}_{24} \mathrm{O}_{9} \mathrm{~N}_{2}, \mathrm{C}_{3} \mathrm{H}_{5} \mathrm{O}_{3}\right)$. The material synthesis procedure was adapted from that of Pluronic P123 templated silica synthesized at neutral $\mathrm{pH}^{[30]}$. Typically, $8.0 \cdot 10^{-2} \mathrm{~g}$ of OCL is mixed with $8.8 \cdot 10^{-2} \mathrm{~g}$ of PEO-b-PMAA in $9.5 \mathrm{~g}$ of deionised water at $T=25^{\circ} \mathrm{C}$. Equilibrium $\mathrm{pH}$ is about 4.3 and it is immediately reached. 0.319 $\mathrm{ml}$ of TEOS is then added to the stirred solution. An emulsion forms and the solution $\mathrm{pH}$ is lowered to about 2. When TEOS hydrolysis is completed (after about 15 minutes under vigorous stirring), the solution $\mathrm{pH}$ is brought to a $\mathrm{pH}$ between 4.5 and 6.5. A precipitate is observed after 20 minutes, the solution is left under stirring for 24 hours at $25^{\circ} \mathrm{C}$. In general, the as-synthesized sample is filtered and placed in an oven at $T=60^{\circ} \mathrm{C}$ overnight. Powder post-synthesis treatment occurs by raising solution $\mathrm{pH}$ to 8 during $48 \mathrm{~h}$ at $25^{\circ} \mathrm{C}$ with the aim to wash the sample for structuring agent removal. This action can take place after powder filtration or in situ in the synthesis batch, just after the synthesis step. The treated material is eventually filtered and dried at $60^{\circ} \mathrm{C}$ overnight. TEOS can then be added again to the leftover solution obtained after washing, if a second synthesis cycle is desired.

Synthesis conditions of silica based materials structured with micelles containing PEO-b-PAA are very similar to those with PEO-b-PMAA polymers. The washing step is somewhat different; therefore, a description of these experiments is given in the supporting information.

\section{Study of the micelle formation as a function of $\mathrm{pH}$ by $\mathrm{DLS}$}

A solution of copolymer was mixed with a solution of oligochitosane. The pH of the mixture was adjusted to a value of 2. The mixture was then titrated by adding small amounts of a $0.5 \mathrm{M} \mathrm{NaOH}$ solution: the $\mathrm{pH}$ was thus progressively increased up to a final value close to 11.5. DLS measurements were done on all the mixtures at varying $\mathrm{pH}$, after each addition of base and after vigorous stirring during five minutes. 


\section{Characterizations}

Dynamic light scattering measurements were carried out with an Autosizer 4800 instrument (Malvern, U.K.) with a $50 \mathrm{~mW}$ laser source operating at $532 \mathrm{~nm}$. Light scattered intensities together with hydrodynamic diameters and polydispersity indexes (PDI) of the micellar colloids were collected. Hydrodynamic diameters were obtained from measured diffusion coefficients by using the Stokes-Einstein equation. The solid mesostructures were studied by SAXS and TEM. SAXS measurements were performed in transmission configuration using a turning copper anode (4 $\mathrm{kW}$ ) as X-ray source with a multilayer focusing “Osmic” monochromator giving high flux $\left(10^{8}\right.$ photons $\left./ \mathrm{sec}\right)$ and punctual collimation was employed. An "Image plate” $2 \mathrm{D}$ detector was used. TEM images were collected on a JEOL 1200 EX II microscope on microtomed samples. Thermogravimetric analysis was performed under air on a TG-DSC 111 Setaram instrument and the temperature ramp was set at $10^{\circ} \mathrm{C} / \mathrm{min}$ up to $1000^{\circ} \mathrm{C} . \mathrm{N}_{2}$ adsorption/desorption experiments

were performed on a Micrometrics Asap 2010 instrument. Solid-state ${ }^{29}$ Si MAS NMR experiments were performed on a Bruker Advance spectrometer $\left(B_{0}=9.4 \mathrm{~T}\right)$ using a $4 \mathrm{~mm}$ zirconia rotor. 


\section{Figure legends :}

Figure 1: Scheme illustrating the strategy for structuring silica by using induced micelles of water soluble block copolymers. Porosity is created at room temperature while the structuring agent is recycled in aqueous solution.

Figure 2: Variation of the scattered light intensity of the PEO-b-PAA/OCL suspension as a function of $\mathrm{pH}$ revealing the formation of micellar aggregates between $\mathrm{pH} 4.5$ and 6.5 and the polymer disassembly out of this $\mathrm{pH}$ domain.

Figure 3: Transmission Electron Microscopy (TEM) images of structured silica obtained with the PEO-b-PMAA/OCL complex at (a) $\mathrm{pH}=4.5$ and (b) $\mathrm{pH}=6.5$ and with the PEO-b-PAA/OCL complex at $\mathrm{pH}=5.5$ (c). Images on top left corner are Fourier Transforms of TEM images. (d) SAXS pattern of the as-synthesized material prepared with micelles of PEO-b-PAA and OCL at $\mathrm{pH}=5.5$.

Figure 4: $\mathrm{N}_{2}$ adsorption/desorption isotherms of ordered mesoporous materials prepared with the PEO-b-PAA/OCL system (synthesis at $\mathrm{pH}=5.5$ ): Material washed ex situ in an aqueous solution at $\mathrm{pH} 8$ (black squares), and corresponding material calcined at 893K without washing (open circles).

Figure 5: TEM images and SAXS curves of as-synthesized sample S1, washed sample S1w and washed sample S1wR (prepared from recycled polymers) obtained with the PEO-b-PMAA/OCL complex system. S1 was obtained at pH 4.5 while powders S1w and S1wR were obtained after an in situ washing treatment at $\mathrm{pH}=8$. 


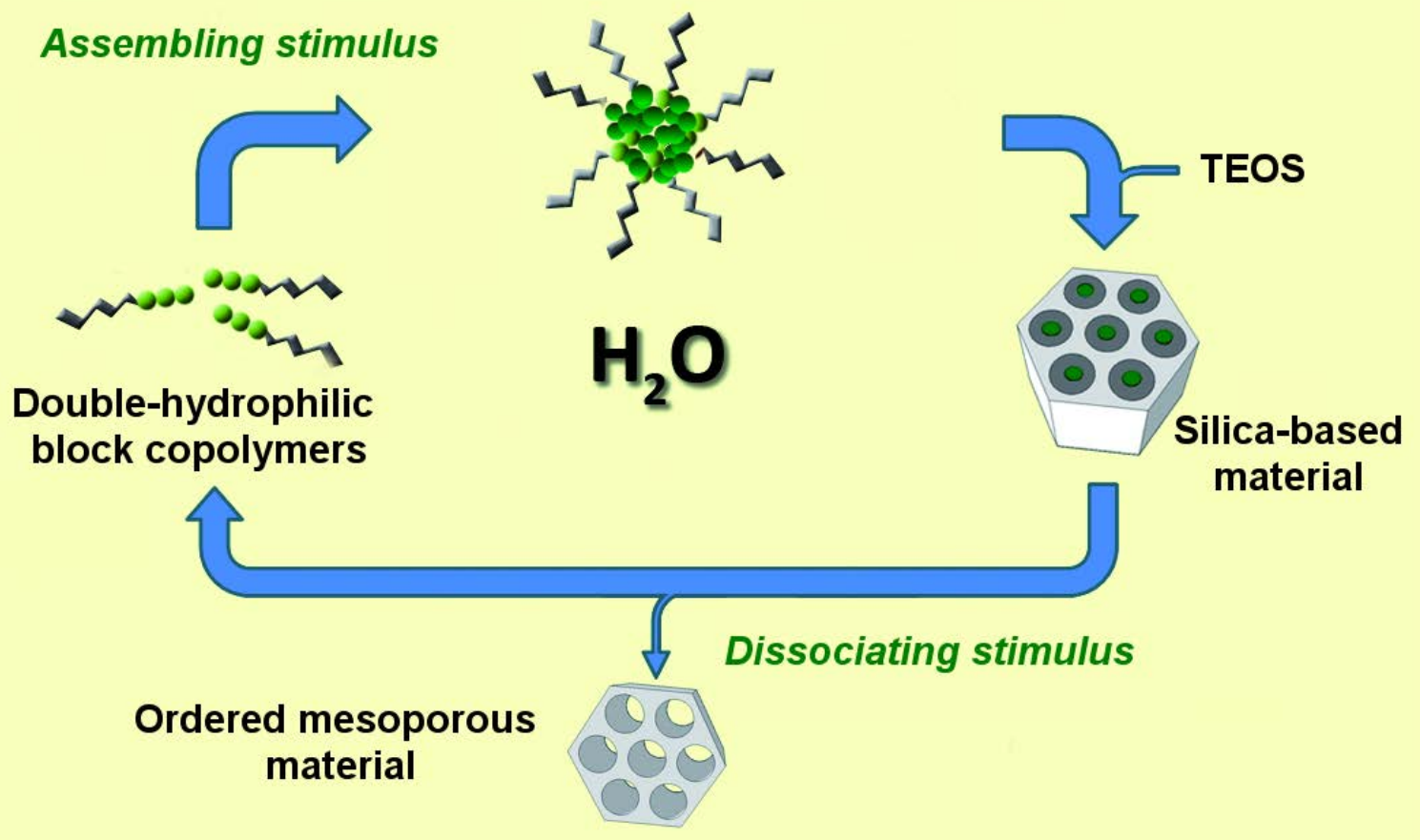

Figure 1 


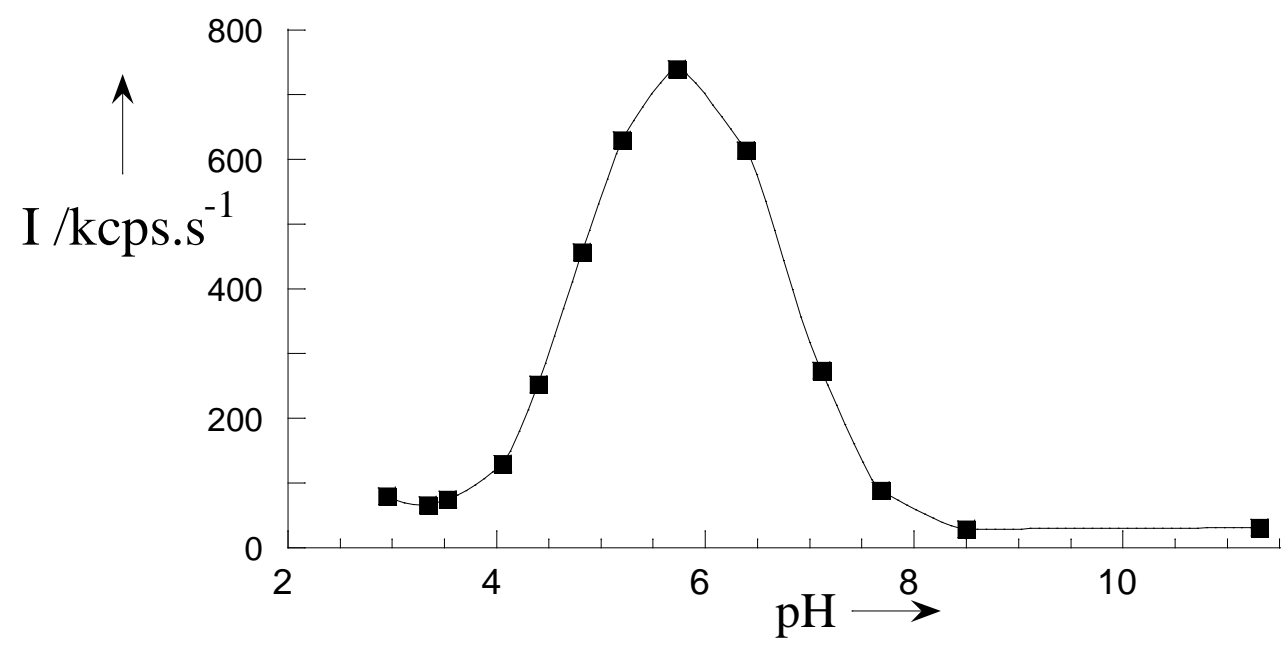

Figure 2 
PEO-b-PMAA/OCL $\mathrm{pH}=\mathbf{4 , 5}$

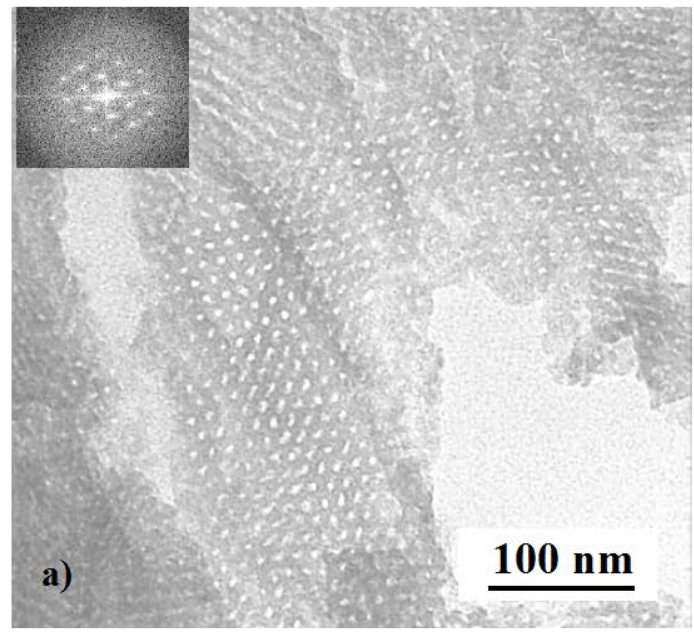

PEO-b-PMAA/OCL $\mathrm{pH}=6,5$

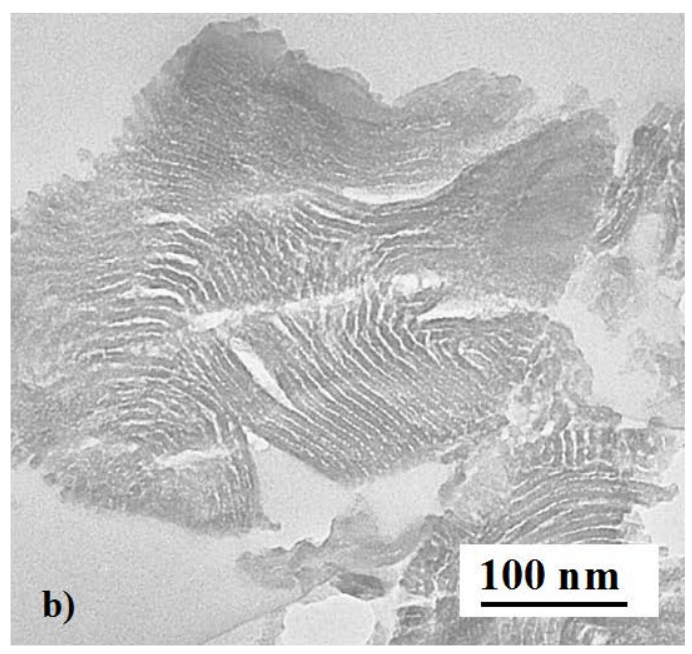

PEO-b-PAA/OCL $p H=5.5$
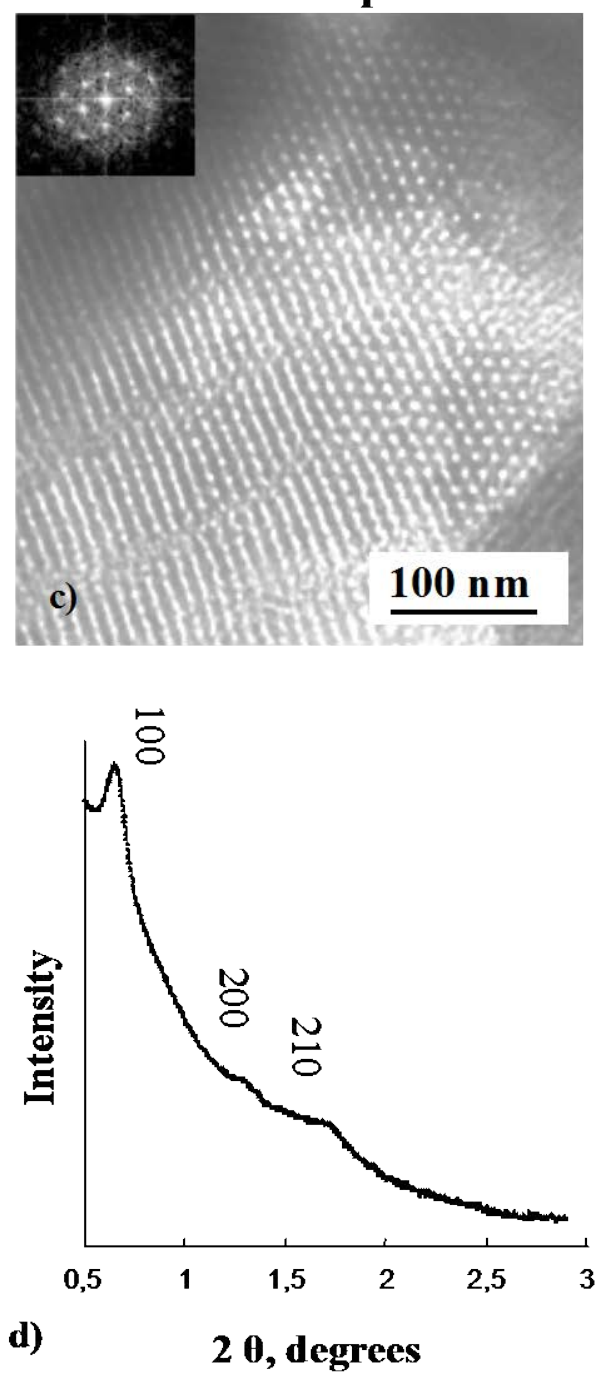

Figure 3 


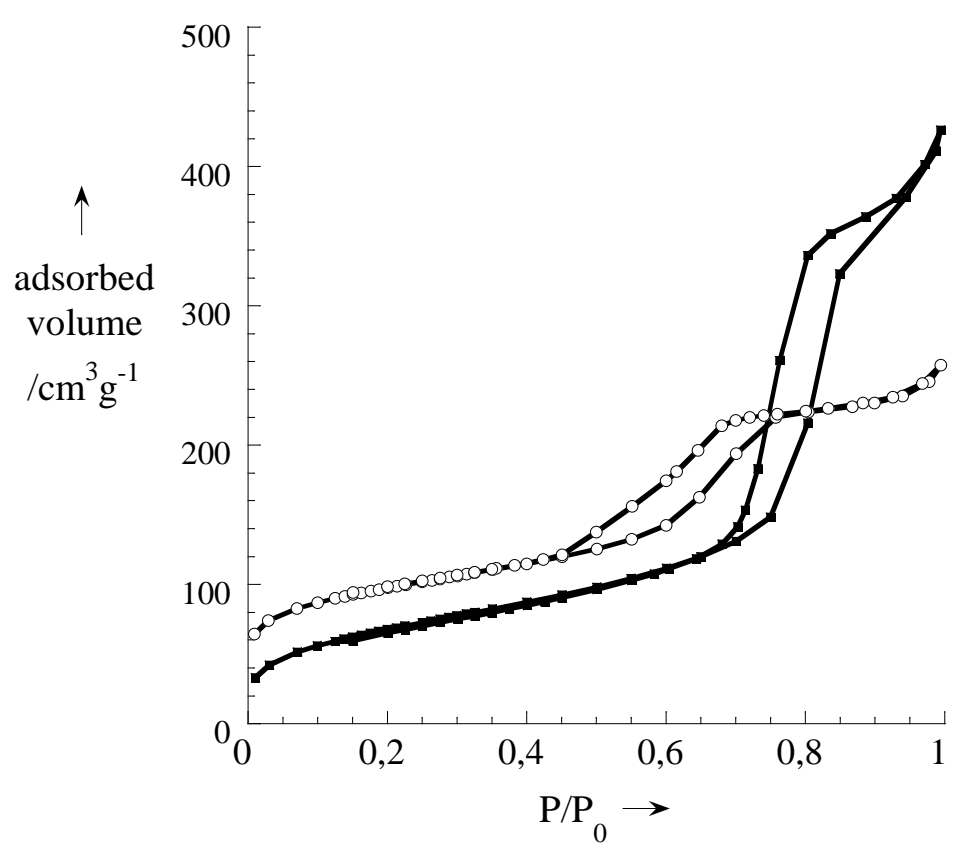

Figure 4 

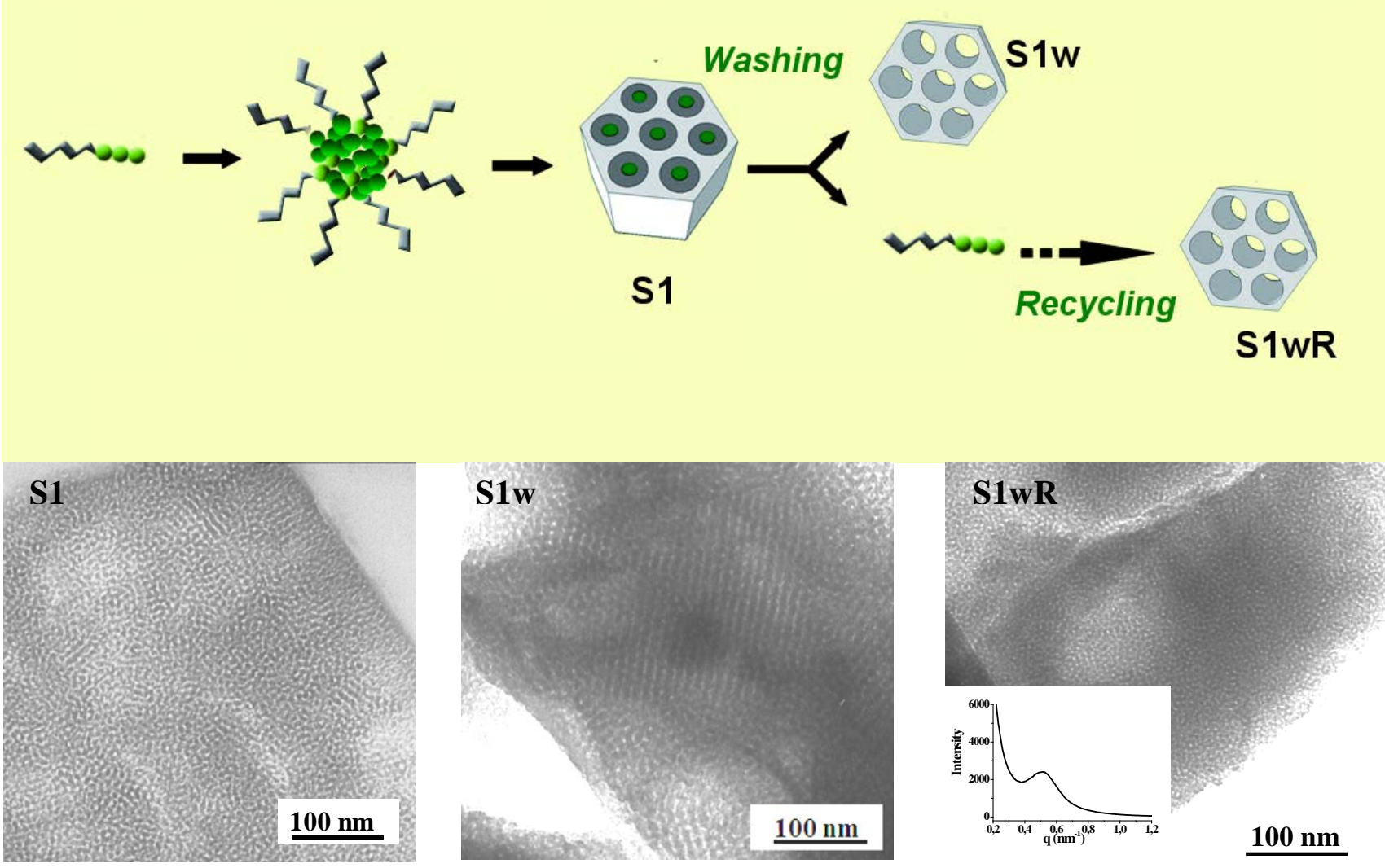

S1 : hybrid material

S1w : washed material (first synthesis)

S1wR : washed material prepared with recycled polymers (second synthesis) 


\section{Short text for the table of contents :}

A new methodology is developed for the synthesis of ordered mesoporous materials in water at room temperature; it relies on the use of reversible and induced micelles of water-soluble block copolymers as new structure directing agents.

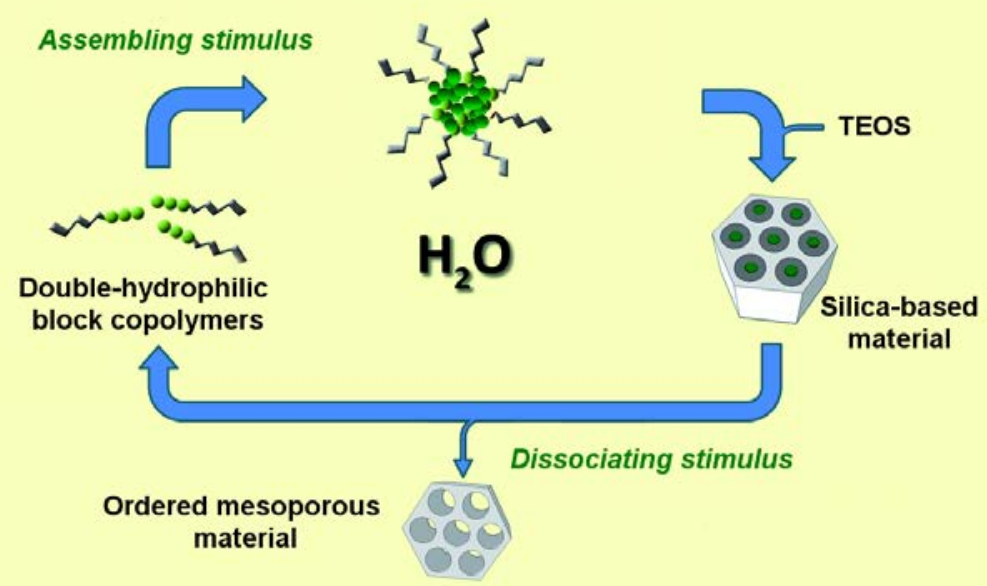

Keywords : block copolymers, mesoporous materials, reversible micelles, polyion complex micelles, silica. 


\section{References :}

[1] C.T. Kresge, M.E. Leonowicz, W.J. Roth, J.C. Vartuli, J.S. Beck, Nature 1992, 359, 710.

[2] Q. Huo, D. I. Margolese, U. Clesla, P. Feng, T. E. Gier, P. Sieger, R. Leon, P. M. Petroff, F. Schuth, G. D. Stucky, Nature 1994, 368, 317.

[3] S.A. Bagshaw, E. Prouzet, T.J. Pinnavaia, Science 1995, 269, 1242.

[4] D. Zhao, J. Feng, Q. Huo, N. Melosh, G. H. Fredrickson, B. F. Chmelka, G. D. Stucky, Science 1998, $279,548$.

[5] Y. Wan, D.Y. Zhao, Chem. Rev. 2007, 107, 2821.

[6] G.J. de A.A. Soler-Illia, C. Sanchez, B. Lebeau, J. Patarin, Chem. Rev. 2002, 102, 4093.

[7] "INORGPOR", GROWTH, G5RD-CT-2000-00317

[8] D. Zhao, Q. Huo, J. Feng, B. F. Chmelka, and G. D. Stucky J. Am. Chem. Soc. 1998, 120 (24), 6024.

[9] M. Benjelloun, P. Van Der Voort, P. Cool, O. Collart, E.F. Vansant, Phys. Chem. Chem. Phys. 2001, 3, 127.

[10] M. Kruk, M. Jaroniec, C.H. Ko, R. Ryoo, Chem. Mater. 2000, 12, 1961.

[11] M.T.J. Keen, R. Denoyel, P.L. Llewellyn, Chem. Commun. 1998, 2203.

[12] S. Kawi, M.W. Lai, Chem. Tech. 1998, 28, 26.

[13] R. Zaleski, J. Wawryszczuk, J. Goworek, A. Borowka, T. Goworek, J. Coll. Interf. Sci. 2003, $262,466$.

[14] H. Lee, S.I. Zones, M. Davis, Nature 2003, 425, 385.

[15] A. Corma, F. Rey, J. Rius, M.J. Sabater, S. Valencia, Nature 2004, 43, 287.

[16] C.C. Pantazis, P.N. Trikalitis, P.J. Pomonis, M.J. Hudson, Micro. Meso. Mater. 2003, 66, 37.

[17] C.C. Pantazis, P.J. Pomonis, Chem. Mater. 2003, 15, 2299.

[18] L. Qi, J. Mater. Sci. Lett. 2001, 20, 2153.

[19] T.M. Long et al., Langmuir 2005, 21, 9365.

[20] D. Eagland, Solution properties of water-soluble polymers, Water Sci. Rev. 4, Cambridge University Press, Cambrige, 1989.

[21] H. Coelfen, M. Antonietti, Langmuir 1998, 14, 582.

[22] H. Coelfen, Macromol. Rapid Comm. 2001, 22, 219.

[23] G. Riess, Prog. Polym. Sci. 2003, 28, 1107.

[24] C. Gu, D. Chen, M. Jiang, Macromol. 2004, 37, 1666.

[25] Y. Li, L.-J. Ding, H. Nakamura, K. Nakashima, J. Coll. Interf. Sci. 2003, 264, 561.

[26] A. Harada, K. Kataoka, Macromol. 1995, 28, 5294. 
[27] A.V. Kabanov, T.K. Bronich, V.A. Kabanov, K. Yu, A. Eisenberg, Macromol. 1996, 29, 6797.

[28] J.-F. Gohy, S.K. Varshney, R. Jérome, Macromol. 2001, 34, 3361.

[29] S. Van der Burgh, A. De Kaizer, M.A. Cohen Stuart, Langmuir 2004, 20, 1073.

[30] K. Matyjaszewski, J. Spanswick, Materials Today, 2005, 26-33.

[31] G. David, C. Boyer, J. Tonnar, B. Ameduri, P. Lacroix-Desmazes, B. Boutevin, Chem. Rev. 2006, 106, 3936.

[32] N. V. Tsarevsky, K. Matyjaszewski, Chem. Rev. 2007, 107, 2270. 